\title{
Implicit versus explicit associative learning and experimentally induced placebo hypoalgesia
}

This article was published in the following Dove Press journal:

Journal of Pain Research

I4 February 20II

Number of times this article has been viewed

\author{
Andrea L Martin-Pichora ${ }^{1,2}$ \\ Tsipora D. Mankovsky-Arnold ${ }^{3}$ \\ Joel Katz' \\ 'Department of Psychology, York \\ University, Toronto, ON, Canada; \\ ${ }^{2}$ Centre for Student Development \\ and Counseling, Ryerson University, \\ Toronto, ON, Canada; ${ }^{3}$ Department \\ of Psychology, McGill University, \\ Montreal, QC, Canada
}

\begin{abstract}
The present study examined whether 1) placebo hypoalgesia can be generated through implicit associative learning (ie, conditioning in the absence of conscious awareness) and 2) the magnitude of placebo hypoalgesia changes when expectations about pain are made explicit. The temperature of heat pain stimuli was surreptitiously lowered during conditioning trials for the placebo cream and the magnitude of the placebo effect was assessed during a subsequent set of trials when the temperature was the same for both placebo and control conditions. To assess whether placebo hypoalgesia could be generated from an implicit tactile stimulus, a $2 \times 2$ design was used with direction of cream application as one factor and verbal information about which cream was being applied as the second factor. A significant placebo effect was observed when participants received verbal information about which cream was being applied but not following implicit conditioning alone. However, $87.5 \%$ of those who showed a placebo response as the result of implicit conditioning were able to accurately guess the order of cream application during the final trial, despite a lack of awareness about the sensory manipulation and low confidence in their ratings, suggesting implicit learning in some participants. In summary, implicit associative learning was evident in some participants but it was not sufficient to produce a placebo effect suggesting some level of explicit expectation or cognitive mediation may be necessary. Notably, the placebo response was abolished when expectations were made explicit, suggesting a delicate interplay between attention and expectation.
\end{abstract}

Keywords: placebo hypoalgesia, associative learning, expectancy, implicit learning

\section{Introduction}

Placebo research has shifted recently from a focus on the inert nature of placebos to examining the contextual factors surrounding a given treatment and the impact of this context on brain-body changes and the subjective experience of the recipient. ${ }^{1}$ Contextual factors contributing to the placebo response include any verbal, visual, auditory, olfactory, or tactile cue, leading an individual to the knowledge/belief that they are receiving a therapeutic treatment. ${ }^{2-4}$

Much of the research on placebo hypoalgesia has examined the impact of verbal cues. Verbal information can influence an individual's expectations about treatment effects and therefore plays a powerful role in shaping both the magnitude ${ }^{5-7}$ and direction ${ }^{8,9}$ of the placebo response. Along with verbal information, expectations can also be acquired through associative learning. Voudouris and colleagues ${ }^{10}$ developed an experimental paradigm whereby the intensity of painful stimuli, delivered to the skin where a placebo cream has been applied, is surreptitiously lowered during a series of conditioning trials, in order to give the impression of analgesic efficacy.
Correspondence: Joel Katz Department of Psychology, York University, Behavioral Sciences Building, 4700 Keele Street, Toronto, ON M3J IP3, Canada Tel 4|6-736-2। 00 ext 40557

Fax 4I6-736-58|4

Email jkatz@yorku.ca 
The placebo response is measured during postconditioning test trials when the intensity of the painful stimulus is readministered at the higher baseline level.

Voudouris and colleagues demonstrated that associative learning can shape placebo effects ${ }^{11}$ and that, under certain circumstances, these effects may be more powerful than those of verbal information. ${ }^{10}$ Conscious expectations have since been shown to play a critical mediating role, ${ }^{12-14}$ suggesting participants learn, through conditioning, that the 'analgesic' agent reduces pain, and thus they develop an expectation that subsequent administration will also lead to less pain. ${ }^{15}$

These studies suggest that conscious expectations play an important role in shaping the placebo response acquired through associative learning. There is, however, some evidence to suggest that associative learning can occur in the absence of explicit expectations. For example, patients conditioned with buprenorphine, a partial mu opioid agonist, exhibit respiratory depression, a side effect of buprenorphine that, while measurable, is not perceptible to patients when given a placebo in the guise of this drug. ${ }^{16,17}$

Research has yet to investigate the potential effects of implicit associative learning on placebo hypoalgesia. As such, the first objective of the present study was to evaluate whether a placebo hypoalgesic effect can be generated by means of implicit associative learning, that is, learning beyond subjective awareness, and thus in the absence of conscious expectations, and if so, to examine whether implicit associative learning enhances the magnitude of the placebo effect when combined with explicit associative learning.

Furthermore, research examining the role of expectations in the placebo response typically relies on subjective reports of expected pain intensity with and without the placebo cream. ${ }^{10,13,14,18,19}$ However, a control group not asked to give a verbal expectancy rating is often not included. Therefore, the second objective of the present study was to examine the impact on pain ratings and the magnitude of the placebo effect of asking subjects to make their expectations about pain explicit.

\section{Methods}

\section{Participants}

Participants comprised 75 adults (51 female and 24 male; mean age $=22.80$, standard deviation $(\mathrm{SD})=5.47$ years $)$ recruited from flyers posted around the university campus. Prior to participating in the study, individuals underwent an initial phone screen to rule out any medical conditions or medication use that might interfere with pain sensitivity or increase risk of unnecessary discomfort during thermal testing. These medical conditions and medications included any ongoing pain problem, high blood pressure, circulatory problems, diabetes, heart disease, asthma, seizures, frostbite, past trauma to the hands or arms, lupus, other large or small joint disease or injury, or current use of analgesics, anti-inflammatory medications, psychoactive drugs, and/or antihistamines. The York University Research Ethics Board reviewed and approved the study protocol. Participants received CAD \$20 for their participation.

\section{Experimental setting and stimuli}

The experiment took place on campus in a room, set up to resemble a doctor's office, with medical equipment, including an examination table, privacy curtain, medical scale for measuring height and weight, blood pressure cuff, metal equipment tray, containers of cotton balls, tongue depressors, plastic syringes, rubbing alcohol, and wall posters depicting the musculoskeletal system, symptoms of neuropathic pain, and the pathophysiology and anatomy of arthritis and knee injury.

Heat pain was induced by means of a Medoc TSA-II thermal stimulator (Medoc Limited, Ramat Yishai, Israel). The TSA-II is a computerized device designed to measure sensory thresholds to vibration and temperature (eg, warm, cold, heat-induced pain, and cold-induced pain). The TSA-II is used in a variety of clinical disorders (eg, diabetes, peripheral neuropathy) to obtain a quantitative evaluation of the integrity of both small- (A-delta and C) and large-diameter (A-beta) sensory nerve fibers. The TSA-II is capable of delivering thermal stimuli that range from $\sim 0^{\circ} \mathrm{C}$ to $50^{\circ} \mathrm{C}$. A thermode is attached to the participant's skin with a Velcro strap, and heat stimuli of various temperatures are administered. A participant-initiated button press stops the rise in temperature, and the thermode rapidly returns to room temperature at a rate of $\sim 4^{\circ} \mathrm{C} / \mathrm{sec}$. Given the relatively brief and limited number of stimuli used in the present study design, even at the highest $\left(49^{\circ} \mathrm{C}\right)$ temperature used, the thermode does not damage the skin although participants may temporarily feel sensitive in the area where the thermode is applied. In the present study, thermal stimuli of 5-sec duration were applied using a thermode with a contact area of $3 \mathrm{~cm}^{2}$. The temperature of the thermode rose rapidly $\left(4^{\circ} \mathrm{C} / \mathrm{sec}\right)$ from a baseline temperature of $35^{\circ} \mathrm{C}$ to a preprogrammed peak temperature where it remained for $5 \mathrm{sec}$ before returning to baseline.

Participants were told that the investigators were studying the effectiveness of a new topical anesthetic cream, called Alevocaine $^{\mathrm{TM}}$, for the purposes of this study, which had been shown to reduce pain in some individuals, and that the 
Alevocaine ${ }^{\mathrm{TM}}$ cream would be compared with a regular moisturizer cream. The creams were visible in two plastic syringes on a metal medical tray with the labels 'Alevocaine ${ }^{\mathrm{TM}}$ ' and 'Control Cream'. The experimenter wore latex gloves while handling and applying the creams, and the creams were removed with an alcohol swab. In actual fact, each container held the same cream, an over-the-counter hypoallergenic moisturizer (Glaxal Base), which did not contain an active analgesic agent. The true nature of these creams was not revealed to participants until the end of the study.

\section{Response measures}

Participants were asked to rate the intensity of pain stimuli using an 11-point self-report Numeric Rating Scale (NRS) ${ }^{20}$ ranging from 0 to 10 , with endpoints representing no pain (0) and the most intense pain imaginable (10). Participants were asked to choose the number that best corresponded to the intensity of each heat pain stimulus they received. The NRS provides a simple, efficient, and minimally intrusive measure of pain intensity. This scale is commonly used in clinical settings ${ }^{21}$ and is the preferred pain rating scale among patients. $^{22}$ The NRS is highly correlated ( $r=0.94$ ) with the visual analog scale ${ }^{21}$ and is sensitive to change following pharmacological interventions. ${ }^{20}$

\section{Procedure}

An experimenter wearing a white lab coat greeted participants upon arrival for the study. Participants were provided with a consent form, and the experimenter described the study following a standard script. Participants were told the investigators were examining the effectiveness of a new, short-acting, local anesthetic called Alevocaine ${ }^{\mathrm{TM}}$ which had been shown to lessen pain in some individuals. The details of the thermal stimulator and the method of assessing the effectiveness of the cream by means of painful heat stimuli were described and participants were told that they could discontinue participation in the study at any time, without negative consequences.

Participants were randomly assigned to one of the five groups according to a predetermined randomization schedule: 1) Direction, 2) No Verbal + No Direction, 3) Verbal + Direction, 4) Verbal, and 5) Verbal + Expectation (the Experimental group section decribes these groups in detail). Similar to the methodology used by Price and colleagues, ${ }^{14}$ each of the experimental groups underwent four stages of thermal testing involving a set of familiarization trials, calibration trials, conditioning trials, and test trials (Figure 1).

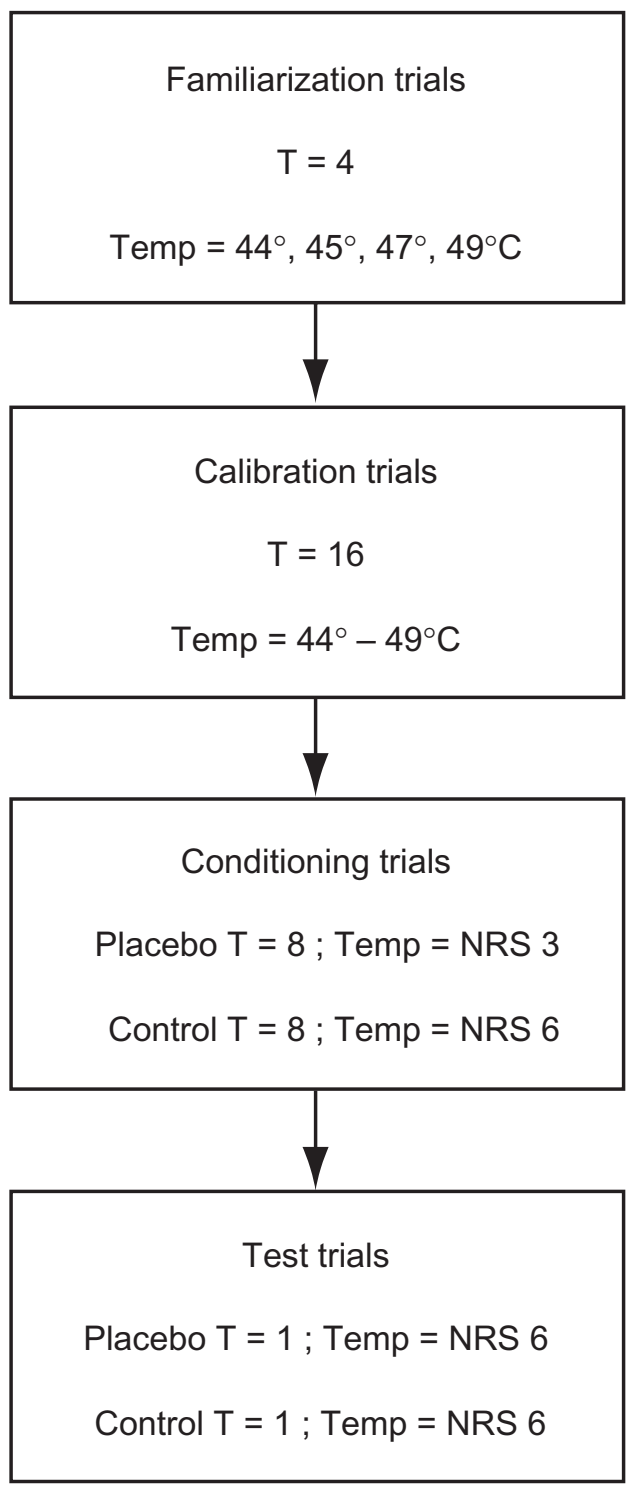

Figure I Flowchart of experimental procedures.

Abbreviations: T, number of trials; NRS, numeric rating scale.

\section{Familiarization trials}

In order to familiarize participants with a range of temperatures, one trial each of $44^{\circ} \mathrm{C}, 45^{\circ} \mathrm{C}, 47^{\circ} \mathrm{C}$, and $49^{\circ} \mathrm{C}$ stimuli was delivered in ascending order on the ventral side of the participant's right forearm.

\section{Calibration trials}

Participants then underwent a series of calibration trials, similar to that described by Price and colleagues, ${ }^{14}$ to control for individual differences in pain perception. A series of 16 thermal stimuli ranging between $44^{\circ} \mathrm{C}$ and $49^{\circ} \mathrm{C}$ was administered in a random order, and participants were asked to rate the pain intensity of each stimulus on a $0-10 \mathrm{NRS}$. At the end of the calibration trials, a regression equation was 
calculated for each participant in order to predict thermal intensity (temperature in ${ }^{\circ} \mathrm{C}$ ) from verbal pain intensity report (NRS pain ratings). This calculation was used to determine the temperature corresponding to each individual's NRS pain rating of 6 and 3, which varied among participants depending on their own personal perception of pain. These two stimulus levels (ie, temperatures) were used in all subsequent trials and were specific to each individual.

\section{Conditioning trials}

The experimenter placed a plastic template on the ventral side of the participant's right forearm and traced two squares on the skin using a marker containing nonpermanent ink. Two square adhesive patches with the center cut out were applied over the two demarcated squares on the forearm to identify where the two creams were to be applied. The creams were applied prior to each conditioning trial.

In line with previous research, ${ }^{10,11,13,23}$ a conditioning procedure was used in which the intensity of heat pain stimuli was surreptitiously lowered during conditioning trials for the placebo cream (ie, Alevocaine ${ }^{\mathrm{TM}}$ cream). That is, in order to create the impression of analgesic efficacy (ie, pain relief) when testing in the area of skin where the placebo cream was applied, the temperature of the heat pain stimulus was surreptitiously lowered to a level corresponding to the participant's NRS pain rating of 3 . When testing in the area of skin where the control cream was applied, the heat pain stimulus was administered at a temperature corresponding to the participant's NRS pain rating of 6. Participants were asked to verbally rate the intensity of each stimulus using the 0-10 NRS.

One block of four thermal stimuli was administered for each cream at each of the two locations according to a randomized counterbalanced design, such that each participant received eight conditioning trials for each cream. The creams were applied prior to each conditioning trial, according to a randomized counterbalanced design since a consistent order of presentation would produce a salient cue signaling which cream was being applied, and thus confound interpretation of the implicit associative learning paradigm.

\section{Test trials}

Immediately following the conditioning trials, participants received one final test trial with each cream. The order of cream application continued to follow a randomized counterbalanced design to ensure that the direction of cream application was the only cue signaling which cream was being applied. Unlike the conditioning trials, the stimulus intensity for the test trials was the same for both the placebo (Alevocaine ${ }^{\mathrm{TM}}$ ) and the control cream. That is, for the 'Alevocaine ${ }^{\mathrm{TM}}$ ' test trial, the stimulus intensity was raised to a temperature corresponding to an NRS pain rating of 6 . The magnitude of the placebo hypoalgesic effect was determined by comparing test trial pain ratings for the placebo versus the control cream.

\section{Experimental groups}

Participants were randomly assigned to one of the five groups: 1) Direction, 2) No Verbal + No Direction, 3) Verbal + Direction, 4) Verbal, and 5) Verbal + Expectation. The details of each experimental group and their relation to the study objectives are described in the following sections.

\section{Implicit associative learning and the placebo effect}

The first objective of the present study was to examine whether it is possible to generate a placebo effect through implicit associative learning; that is, in response to a conditioning procedure in which the conditioned stimulus, a tactile cue involving direction of cream application, is established in the absence of the participant's awareness. In order to test the first objective, a $2 \times 2$ design was employed, with direction of cream application (Direction) as one factor and verbal information about which cream was being applied (Verbal) as the second factor (Figure 2).

The direction of cream application was manipulated for the Direction group, such that unbeknown to the participants, the placebo cream (Alevocaine ${ }^{\mathrm{TM}}$ ) was always applied using

\section{Directional application}

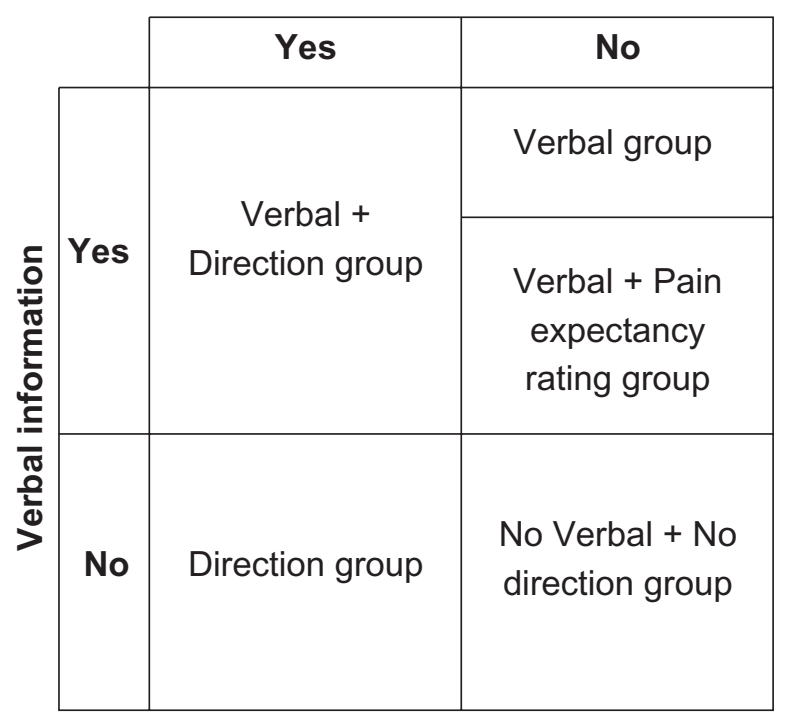

Figure 2 A $2 \times 2$ design showing the direction of application and verbal information factors depicting the five experimental groups. 
10 upward strokes and the 'control cream' was always applied using 10 downward strokes. Throughout the conditioning and test trials, participants in the Direction group were not verbally told which cream was Alevocaine ${ }^{\mathrm{TM}}$ and which was the 'control'; the only cue was the direction of cream application. The Direction group was used to determine whether a placebo effect could be generated from a tactile stimulus, of which participants are unaware (ie, the direction of cream application). To ensure that the placebo responses observed in the Direction group were a function of the tactile directional manipulation, a No Verbal + No Direction condition was included as a control group. The No Verbal + No Direction group was not told which cream was being applied before conditioning and test trials and did not receive the tactile directional manipulation (application of the placebo cream (Alevocaine ${ }^{\mathrm{TM}}$ ) in one direction and the control cream in the other direction); instead, both creams were applied in a circular motion.

In order to evaluate the relative contribution of explicit associative learning and implicit associative learning to the magnitude of the placebo response, a Verbal group was included in the study design. The Verbal group was verbally told which cream was being applied before the conditioning and test trials, thus reinforcing a conscious expectation for pain relief with Alevocaine ${ }^{\mathrm{TM}}$ application. Placebo effects generated from the conditioning procedure in this group would likely be mediated by explicit learning, since participants were consciously aware of when the 'active' treatment was being applied. This group did not receive the tactile directional manipulation; instead, both creams were applied in a circular motion (similar to the No Verbal + No Direction group).

A Verbal + Direction group was also included to test whether the magnitude of the placebo effect changes when explicit associative learning is combined with implicit associative learning. The Verbal + Direction group was told which cream was being applied before the conditioning and test trials (similar to the Verbal group) and received the tactile directional manipulation (similar to the Direction group).

\section{Explicit expectations and the placebo effect}

The second objective of the present study was to investigate the effect of asking participants, just prior to the test trial with each cream, to rate the pain intensity they expected to experience in response to the ensuing heat pain stimulus. As such, a fifth group, the Verbal + Expectation group, received the same treatment as the Verbal group, except that following the conditioning trials, just prior to the test trial with each cream, participants were asked to rate the pain intensity they expected to experience in response to the imminent heat pain stimulus delivered to the Alevocaine ${ }^{\mathrm{TM}}$ and control cream-treated skin.

\section{Test of implicit associative learning and posttest interview}

Following the thermal testing with the creams, all participants were asked to rate the intensity and unpleasantness of light touch applied to the area of skin where the creams had been tested. The purpose of these sensory tests was to obtain an implicit measure of associative learning in the Direction group. The light touch was applied by the experimenter using 10 upward strokes of the index finger, in exactly the same manner as the placebo cream had been applied for the Direction group during the conditioning and test trials and again using 10 downward strokes of the index finger, in exactly the same manner as the control cream had been applied for the Direction group during the conditioning and test trials.

Participants were asked to rate the intensity and unpleasantness of these tactile stimuli using a NRS. The NRS for intensity ranged from not at all intense (0) to the most intense sensation imaginable (10). Similarly, the NRS for unpleasantness ranged from not at all unpleasant (0) to the most unpleasant sensation imaginable (10). This test of implicit learning was similar to that used by Seamon and Delgado. ${ }^{24}$ Provided participants are unaware of the directional manipulation, lower intensity and/or unpleasantness ratings in response to the light stroking of the skin applied in an upward motion (similar to that during application of the placebo cream), compared to stimuli applied in a downward motion (similar to that during application of the control cream), would suggest evidence of implicit associative learning in the Direction group. All study groups underwent this sensory testing to evaluate the hypothesis that differences in NRS intensity and unpleasantness ratings between the upward and downward stroking were a function of the tactile directional manipulation associated with the two creams during conditioning trials in the Direction group.

Following the sensory testing, participants were interviewed about their perceptions of the effectiveness of Alevocaine ${ }^{\mathrm{TM}}$. As a validity check, participants were also questioned in an unbiased manner about the upward and downward direction of application of the two creams in order to ascertain the extent of their awareness of the directional manipulation. At the end of the interview, participants were 
debriefed about the purpose of the study, and the true nature of the creams was explained.

\section{Results}

\section{Effect of implicit associative learning}

A $5 \times 2$ between-within analysis of variance (ANOVA) was conducted to evaluate differences in NRS pain intensity scores for placebo and control creams across the five experimental groups, using group (ie, Direction, No Verbal + No Direction, Verbal + Direction, Verbal, and Verbal + Expectation) as the between subjects factor and cream (Placebo and Control) as the within subjects, repeated measures factor.

ANOVA results revealed a significant main effect for cream (Placebo/Control), $F(1,70)=16.46, P<0.001$, and a significant group $\times$ cream interaction effect, $F(4,70)=3.00$, $P=0.02$. NRS pain scores were significantly lower for the placebo cream $(\mathrm{M} \pm \mathrm{SD}=5.43 \pm 2.16)$ than the control cream $(\mathrm{M} \pm \mathrm{SD}=6.19 \pm 1.99)$. Simple effects of the group $\times$ cream interaction evaluating cream within group showed a significant placebo effect for the Verbal group, $F(1,70)=9.08, P=0.004(\mathrm{M} \pm \mathrm{SD}$ NRS pain scores: placebo $=5.53 \pm 1.85$, control $=6.73 \pm 1.91)$ and the Verbal + Direction group, $F(1,70)=14.83, P<0.001(\mathrm{M} \pm$ SD NRS pain scores: placebo $=5.07 \pm 1.91$, control $=6.60 \pm 1.30$ ) Comparisons between NRS pain scores for placebo versus control creams were not significantly different within the Direction, No Verbal + No Direction, and Verbal + Expectation groups (see Table 1). The main effect for group was not significant, $F(4,70)=0.651, P=0.63$.

Sensory testing and posttest interview data were examined to further explore evidence of implicit learning in the Direction group, despite the lack of a significant difference in pain ratings between the two creams in this group. Paired two-tailed $t$ tests showed no significant difference in intensity $t(14)=0.00, P=1.00(\mathrm{M} \pm \mathrm{SD}$ intensity ratings: upward motion $=1.07 \pm 1.33$, downward motion $=1.07 \pm 1.22)$ or unpleasantness ratings $t(14)=-0.34, P=0.74(\mathrm{M} \pm \mathrm{SD}$ unpleasantness ratings: upward motion $=0.73 \pm 1.28$, downward motion $=0.87 \pm 1.12$ ) between touch administered in an upward motion versus a downward motion for the Direction group during the sensory testing.

However, examination of posttest interview data revealed that when participants in the Direction group were asked to guess which cream had been applied first and which cream had been applied second during the final set of trials, $87.5 \%$ $(7 / 8)$ of those who showed a placebo response were able to accurately identify the order of cream application, compared to only $14 \%(1 / 7)$ of those who did not show a placebo response (Fisher exact test, $P=0.01$ ). When asked to rate how confident they were in their guess, an independent samples $t$ test revealed no significant difference in reported confidence between those who guessed the order of application correctly $(\mathrm{M} \pm \mathrm{SD}=4.63 \pm 2.72)$ and those who guessed incorrectly across the group as a whole $(\mathrm{M} \pm \mathrm{SD}=4.43 \pm 3.46)$, $t(13)=-0.12, P=0.90$.

Results of a validity check revealed that when asked if they noticed that one cream was always applied in an upward motion and one cream was always applied in a downward motion, only $7 \%(1 / 15)$ of participants in the Direction group and $13 \%(2 / 15)$ of participants in the Verbal + Direction group reported being aware of the tactile directional manipulation. ${ }^{\text {a }}$ Two of these participants reported becoming aware of the direction of cream application during the final (test) trial, and the other participant reported noticing the direction of application from the beginning of the testing with the creams.

These results suggest that the majority of subjects were unaware of the direction manipulation, and confidence ratings were the same across all subjects in the direction group (ie, participants believed they were guessing the order of cream application). However, even though the Direction group as a whole did not show a significant placebo effect, individual participants within the Direction group who showed a placebo response (ie, who rated Alevocaine ${ }^{\mathrm{TM}}<$ Control) during the final test trial were significantly more likely to accurately guess the order of cream application than those who did not show a placebo response.

\section{Effect of verbalizing explicit expectations}

Interestingly, the Verbal + Expectation group did not demonstrate a significant placebo effect. A 2 (cream: Placebo/ Control) $\times 2$ (pain rating: Expected/Actual) repeated measures ANOVA was conducted to examine the relationship between participants' expected pain ratings and actual pain ratings during the final test trial. Results revealed a significant main effect for cream, $F(1,14)=7.59, P=0.02$, and a significant pain rating $\times$ cream interaction effect, $F(1,14)=29.52$, $P<0.001$. Simple effects analyses of cream within pain rating showed a significant difference in expected pain rating $(\mathrm{M} \pm \mathrm{SD} \mathrm{NRS}$ pain scores: placebo $=2.86 \pm 2.18$, control $=5.36 \pm 1.99) F(1,14)=17.33, P=0.001$, but not the

${ }^{\mathrm{a}}$ After removing the one participant from the Direction group who reported being aware of the direction of cream application, the proportion of placebo responders who accurately identified the order of cream application (6/7) remained significantly greater than that of nonresponders (1/7), Fisher exact test, $P=0.03$. 
Table I Means (M) and standard deviations (SD) for numeric rating scale pain intensity scores in response to test trials when heat pain stimuli of identical temperatures were delivered to skin treated with placebo (Alevocaine ${ }^{\mathrm{TM}}$ ) and control creams

\begin{tabular}{|c|c|c|c|c|}
\hline \multirow[t]{2}{*}{ Group } & \multicolumn{3}{|l|}{$M(S D)$} & \multirow[t]{2}{*}{$F$ statistic and $P$ value } \\
\hline & Placebo (P) & Control (C) & Placebo effect $(C-P)$ & \\
\hline Verbal & $5.53(1.85)$ & $6.73(1.91)$ & $1.20(1.97)$ & $F(I, 70)=9.08, P=0.004$ \\
\hline Direction & $5.87(2.26)$ & $6.47(1.96)$ & $0.60(1.76)$ & $F(I, 70)=2.27, P=0.14$ \\
\hline Verbal + Direction & $5.07(1.91)$ & $6.60(1.30)$ & $1.53(\mathrm{I} .46)$ & $F(I, 70)=14.83, P<0.001$ \\
\hline No Verbal + No Direction & $5.93(2.12)$ & $5.67(2.26)$ & $-0.27(1.10)$ & $F(I, 70)=0.45, P=0.5 I$ \\
\hline Verbal + Expectation & $4.73(2.63)$ & $5.43(2.31)$ & $0.57(1.22)$ & $F(I, 70)=1.92, P=0.17$ \\
\hline
\end{tabular}

Note: $F$ statistics represent simple effects of the group $\times$ cream interaction evaluating cream within group and show a significant placebo effect for the Verbal and Verbal + Direction groups.

actual pain rating during the final trial (Table 2). That is, just prior to the final trial, participants in the Verbal + Expectation group expected to experience significantly less pain with the placebo cream than with the control cream. However, this difference between the placebo cream and the control cream was not reflected in the actual pain ratings during the final test trial with each of the creams (Figure 3).

Follow-up analyses of variance were conducted to look for differences in pain sensitivity or the effectiveness of the conditioning trials for the Verbal + Expectation group that might account for the lack of a significant placebo effect in this group. Results revealed no significant differences between the Verbal + Expectation group and the other four experimental groups with regard to pain sensitivity, as measured by mean pain intensity ratings across calibration trials $(F(4,74)=1.33, P=0.27)$, mean difference between temperatures corresponding to subjective pain ratings of 6 and $3(F(4,74)=1.03, P=0.40)$, or perceived effectiveness of Alevocaine ${ }^{\mathrm{TM}}$ during conditioning trials, as measured by subtracting the mean pain rating for placebo cream from the mean pain rating for control cream across conditioning trials $(F(4,74)=1.08, P=0.37)($ see Table 3$)$.

\section{Discussion}

The present study examined whether a placebo effect could be generated by means of implicit associative learning using a tactile cue; that is, conditioning in the absence of conscious awareness. A placebo effect, as measured by a significant

Table 2 Means (M) and standard deviations (SD) for expected and actual numeric rating scale pain intensity scores in the Verbal + Expectation group

\begin{tabular}{llll}
\hline & $M(S D)$ & & \\
\cline { 2 - 4 } & Placebo & Control & Control - placebo \\
\hline Expected pain rating & $2.86(2.18)$ & $5.36(1.99)$ & $2.40(1.24)^{*}$ \\
Actual pain rating & $4.86(2.68)$ & $5.43(2.31)$ & $0.57(1.22)$ \\
\hline Notes: $* F(I, I 4)=17.33 ; P=0.001$. & &
\end{tabular}

reduction in pain intensity scores for the placebo relative to the control cream following conditioning trials, was observed when participants received verbal information about which cream was being applied throughout the conditioning and test trials; that is, conditioning by means of explicit associative learning. However, our implicit conditioning manipulation (ie, placebo cream applied in an upward motion and control cream applied in a downward motion) did not generate a significant placebo effect.

We did, nevertheless, find some evidence to suggest learning may have occurred in some participants in the absence of conscious awareness, as a result of our implicit conditioning manipulation. In particular, when participants who received the directional manipulation were asked to

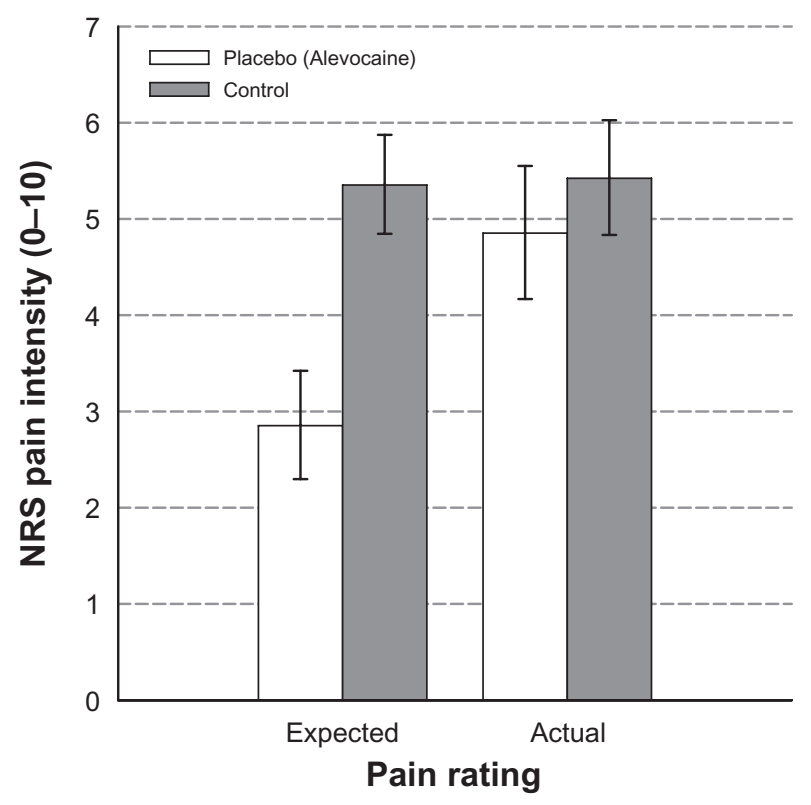

Figure 3 Mean expected and actual numeric rating scale (NRS) pain intensity ratings in response to heat pain stimuli for placebo and control cream test trials in the Verbal + Expectation group. Error bars depict standard error of the mean. Results indicate participants in the Verbal + Expectation group expected to experience significantly less pain with the placebo cream than with the control cream; however, this difference was not reflected in the actual pain ratings. 
Table 3 Group means (M) and standard deviations (SD) for average numeric rating scale (NRS) pain intensity scores across calibration trials, mean difference between heat pain stimulus temperatures corresponding to NRS pain scores of 6 and 3 , and mean difference in average NRS pain intensity scores for control cream minus average pain intensity for placebo cream during conditioning trials

\begin{tabular}{llll}
\hline Group & M (SD) & & \\
\cline { 2 - 4 } & $\begin{array}{l}\text { Mean pain intensity rating } \\
\text { across calibration trials }\end{array}$ & $\begin{array}{l}\text { Mean temperature } \\
\text { for NRS of } 6 \text { minus mean } \\
\text { temperature for NRS of } \mathbf{3}\end{array}$ & $\begin{array}{l}\text { Mean pain control cream minus } \\
\text { mean pain placebo during } \\
\text { conditioning }\end{array}$ \\
\hline Direction & $5.18(1.87)$ & $3.20(1.15)$ & $1.92(1.17)$ \\
No Verbal + No Direction & $4.13(1.65)$ & $3.07(I .44)$ & $2.00(0.93)$ \\
Verbal + Direction & $4.95(I .44)$ & $2.80(0.77)$ & $2.21(1.13)$ \\
Verbal & $4.84(1.12)$ & $2.87(0.99)$ & $2.59(1.16)$ \\
Verbal + Expectation & $3.88(2.85)$ & $3.53(1.13)$ & $1.85(1.14)$ \\
\hline
\end{tabular}

guess the order of cream application during the final trial, $87.5 \%$ of placebo responders guessed accurately, compared to only $14 \%$ of nonresponders. The majority of participants (14 of 15) were unaware of the direction of cream application and confidence ratings, for the order of cream application did not differ between those who guessed correctly and those who guessed incorrectly. Given that the order of cream application was counterbalanced across participants, the significantly higher than chance accuracy of their ratings, combined with participants' reported lack of awareness about the tactile manipulation and low confidence in their ratings (ie, participants believed they were guessing), suggests the possibility of implicit associative learning in some participants; that is, conditioning in the absence of conscious, verbally accessible expectations.

There are several possible explanations for the lack of a significant placebo effect across the Direction group as a whole. It is possible that the conditioned stimulus (ie, the direction of cream application) was too subtle to be detected by the participants' sensory perceptual apparatus or may have lacked the relevance needed to be processed either explicitly or implicitly. Previous research has examined conditioned responding to a tactile stimulus (eg, sandpaper) in rats ${ }^{25,26}$ and a conditioned head turn response in neonates following pairing of a sound with a stroke to the cheek. ${ }^{27}$ However, to our knowledge, this is the first study of its kind attempting to condition a placebo effect to a tactile stimulus, and it is possible that the direction of cream application may have lacked the informational value necessary to be identified as a reliable and unique signal for the occurrence of reduced pain.

The second possibility is that the effects of conditioning on placebo hypoalgesia are indeed mediated by conscious expectations, as has been argued by numerous researchers in the field. ${ }^{12-14}$ De Jong and colleagues ${ }^{12}$ used a conditioning procedure similar to that of the present study and found a correlation between expected and actual level of hypoalgesia across all groups. Furthermore, informing participants that the stimulus intensity was being reduced for the placebo cream resulted in lower expectations for pain relief and reduced placebo responding. Montgomery and Kirsch ${ }^{13}$ replicated these findings and found the placebo hypoalgesic effect disappeared when they controlled for expectancies, suggesting the effect of conditioning on the placebo effect was mediated by explicit expectations generated from verbal information.

Similarly, Price and colleagues ${ }^{14}$ found placebo effects on pain intensity and pain unpleasantness were strongly associated with expectancy for pain relief and like Montgomery and Kirsch ${ }^{13}$ concluded that, '... although conditioning may be sufficient for placebo analgesia, it is likely to be mediated by expectancy' (p. 147). Additionally, studies utilizing an open-hidden paradigm have shown that open administration of a drug is significantly more effective than a hidden administration, ${ }^{28-30}$ indicating treatments are less effective when subjects are unaware of the treatment.

However, the question still remains as to whether all placebo effects are mediated by conscious expectations. The relationship between awareness and associative learning has received much attention in experimental psychology, and several studies provide evidence of conditioned responding without awareness. Schell and colleagues ${ }^{31}$ found skin conductance responses conditioned to phobic stimuli persisted even after participants' cognitive expectancy of the unconditioned stimulus was completely extinguished. Furthermore, conditioning in animals under anesthesia has been observed in numerous studies ${ }^{32-35}$ suggesting evidence for the possibility of unconscious conditioning in humans, although these findings are yet to be replicated in anesthetized human participants. ${ }^{36}$ Additionally, studies of conditioned taste aversion ${ }^{37-39}$ provide compelling evidence 
that conditioning in humans is not always cognitively mediated. Therefore, while some studies point to the important mediating role of explicit expectations in the placebo analgesic response, there is sufficient evidence to suggest that conditioning can occur in the absence of explicit expectations leaving open the possibility that placebo hypoalgesia could be conditioned without explicit expectations (ie, via implicit learning).

As such, the third possible explanation is that some instances of conditioning are mediated by conscious expectations, while others are not. As Lovibond and Shanks ${ }^{40}$ suggest, conditioning without awareness may occur within relatively specialized systems, such as the gustatory system in conditioned taste aversion. Benedetti and colleagues ${ }^{16,17}$ found evidence of a placebo respiratory depression in patients previously treated with buprenorphine, who were then subsequently administered an infusion of saline and told it was buprenorphine, but this effect was not found in an unconditioned control group. These researchers note that while cognitive and affective mechanisms may be involved in placebo analgesia, the mild respiratory depressant effect of buprenorphine goes unnoticed by patients, suggesting that a conditioning mechanism independent of cognition may be involved in accordance with the earlier conditioning hypotheses of Wickramasekera ${ }^{41}$ and Voudouris and colleagues. $^{10,23}$

In another study, Benedetti and colleagues ${ }^{42}$ found that verbally induced expectations of growth hormone $(\mathrm{GH})$ increase or decrease produced no change in plasma concentration levels, except in subjects who received preconditioning with a GH stimulator. In contrast, verbally induced expectations affected pain in healthy subjects and motor performance in individuals with Parkinson's disease. ${ }^{42}$ Therefore, conditioning and expectancy mechanisms may both be involved in the placebo response; conditioning in unconscious processes (eg, hormone secretion and respiratory depression) and expectation in conscious processes (eg, pain and motor performance). ${ }^{42-44}$ Additionally, Benedetti's studies involve pharmacological conditioning in contrast to the present study and others that use a conditioning paradigm without administration of pharmacological agents, and as Stewart-Williams and Podd ${ }^{44}$ point out, this may be a crucial difference. Placebo effects in which there is no previous experience with a pharmacologically active agent may be mediated by explicit expectations whereas placebo effects that are not cognitively mediated may occur when a placebo mimics pharmacologically active substances with which the participant has had previous experience. In the present study, only 31 of 75 participants reported any previous experience with a local anesthetic cream. It would be interesting for future research to explore the present implicit conditioning paradigm in subjects who had been preconditioned with an active analgesic cream. Additionally, incorporating an objective implicit response measure, such as galvanic skin response, would provide additional support for implicit associative learning mechanisms. It is also possible that there is in fact a small effect size for implicit conditioning, and there was not sufficient power in the present study to detect group differences, given the small sample size.

The second objective of the present study was to examine whether asking participants to make their expectations about pain explicit has any impact on pain ratings and the magnitude of the placebo effect. Results indicated a significant difference in expected pain ratings, but not in actual pain ratings during the final placebo trial. The Verbal + Expectation group did not differ from the other groups with respect to their pain sensitivity, mean temperature difference between ratings of 6 and 3, or difference in pain ratings for placebo and control during conditioning trials, suggesting that the differences between expected and actual pain ratings observed in the Verbal + Expectation group were related to the experimental manipulation of asking participants to verbalize their expectations prior to the final trial.

We hypothesized that asking participants to make their expectations about pain intensity explicit might enhance placebo responding due to demand characteristics, a desire to please the experimenter, and/or a desire for consistency between their expectations and subsequent experience (ie, actual pain ratings). It is not clear why the placebo effect was abolished in this group. One possible explanation is that asking participants to rate the amount of pain they expect to experience with each cream just prior to the final trial drew additional attention to their somatosensory experience, thus highlighting the lack of congruence between their expectations and actual experience. It is also possible that the lack of a significant placebo effect in this group is a function of sample size.

In summary, although evidence was adduced to suggest that learning, in the absence of conscious awareness, might have occurred as a result of the implicit conditioning procedure in some participants, implicit associative learning was not sufficient to produce a placebo hypoalgesic effect. This suggests some level of conscious expectation or cognitive mediation may be necessary to generate a placebo 
effect, at least with conditioning paradigms that do not involve administration of pharmacological agents. Notably, the placebo effect was abolished when expectations for pain relief were made explicit, suggesting a delicate interplay exists between attention and expectation.

\section{Acknowledgments}

We thank Eileen Halket for help with data collection and entry. Andrea Martin-Pichora was supported by a Canadian Institutes of Health Research (CIHR) Doctoral Award and a CIHR Strategic Training Fellowship in Pain: Molecules to Community. Joel Katz is supported by a CIHR Canada Research Chair (CRC) in Health Psychology at York University. The study was funded by a CIHR CRC in Health Psychology and by infrastructure grants from the Canadian Foundation for Innovation and the Ontario Innovation Trust. This article is derived, in part, from Andrea Martin-Pichora's Doctoral Dissertation.

\section{Disclosure}

The authors report no conflicts of interest in this work.

\section{References}

1. Colloca L, Benedetti F. Placebos and painkillers: is mind as real as matter? Nat Rev Neurosci. 2005;6(7):545-552.

2. Benedetti F, Amanzio M. The neurobiology of placebo analgesia: from endogenous opioids to cholecystokinin. Prog Neurobiol. 1997; 52(2):109-125.

3. Benedetti F, Pollo A. The pharmacology of placebos. Int J Pain Med Palliative Care. 2001;1:42-48.

4. Benedetti F. How the doctor's words affect the patient's brain. Eval Health Prof. 2002;25(4):369-386.

5. Kirsch I, Rosadino MJ. Do double-blind studies with informed consent yield externally valid results? An empirical test. Psychopharmacology (Berl). 1993;110(4):437-442.

6. Kirsch I, Weixel LJ. Double-blind versus deceptive administration of a placebo. Behav Neurosci. 1988;102(2):319-323.

7. Pollo A, Amanzio M, Arslanian A, Casadio C, Maggi G, Benedetti F. Response expectancies in placebo analgesia and their clinical relevance. Pain. 2001;93(1):77-84.

8. Butler C, Steptoe A. Placebo responses: an experimental study of psychophysiological processes in asthmatic volunteers. Br J Clin Psychol. 1986;25(Pt 3):173-183.

9. Luparello TJ, Leist N, Lourie CH, Sweet P. The interaction of psychologic stimuli and pharmacologic agents on airway reactivity in asthmatic subjects. Psychosom Med. 1970;32(5):509-513.

10. Voudouris NJ, Peck CL, Coleman G. The role of conditioning and verbal expectancy in the placebo response. Pain. 1990;43(1):121-128.

11. Voudouris NJ, Peck CL, Coleman G. Conditioned placebo responses. J Pers Soc Psychol. 1985;48(1):47-53.

12. De Jong PJ, van Baast R, Arntz A, Merckelbach H. The placebo effect in pain reduction: the influence of conditioning experiences and response expectancies. Int J Behav Med. 1996;3(1):14-29.

13. Montgomery GH, Kirsch I. Classical conditioning and the placebo effect. Pain. 1997;72(1-2):107-113.

14. Price DD, Milling LS, Kirsch I, Duff A, Montgomery GH, Nicholls SS. An analysis of factors that contribute to the magnitude of placebo analgesia in an experimental paradigm. Pain. 1999;83(2):147-156.
15. Kirsch I. Specifying nonspecifics: psychological mechanisms of placebo effects. In: Harrington A, editor. The Placebo Effect: An Interdisciplinary Exploration. Cambridge (MA): Harvard University Press; 1997:166-186.

16. Benedetti F, Amanzio M, Baldi S, et al. The specific effects of prior opioid exposure on placebo analgesia and placebo respiratory depression. Pain. 1998;75(2-3):313-319.

17. Benedetti F, Amanzio M, Baldi S, Casadio C, Maggi G. Inducing placebo respiratory depressant responses in humans via opioid receptors. Eur J Neurosci. 1999;11(2):625-631.

18. De Pascalis V, Chiaradia C, Carotenuto E. The contribution of suggestibility and expectation to placebo analgesia phenomenon in an experimental setting. Pain. 2002;96(3):393-402.

19. Vase L, Robinson ME, Verne GN, Price DD. The contributions of suggestion, desire, and expectation to placebo effects in irritable bowel syndrome patients. An empirical investigation. Pain. 2003;105(1-2): $17-25$.

20. Katz J, Melzack R. Measurement of pain. Surg Clin North Am. 1999; 79(2):231-252.

21. Bijur PE, Latimer CT, Gallagher EJ. Validation of a verbally administered numerical rating scale of acute pain for use in the emergency department. Acad Emerg Med. 2003;10(4):390-392.

22. Williamson A, Hoggart B. Pain: a review of three commonly used pain rating scales. J Clin Nurs. 2005;14(7):798-804.

23. Voudouris NJ, Peck CL, Coleman G. Conditioned response models of placebo phenomena: further support. Pain. 1989;38(1):109-116.

24. Seamon JG, Delgado MR. Recognition memory and affective preference for depth-rotated solid objects: part-based structural descriptions may underlie the mere exposure effect. Vis Cogn. 1999;6(2):145-164.

25. Nizhnikov ME, Pautassi RM, Truxell E, Spear NE. Opioid antagonists block the acquisition of ethanol-mediated conditioned tactile preference in infant rats. Alcohol. 2009;43(5):347-358.

26. Molina JC, Bannoura MD, Chotro MG, McKinzie DL, Arnold HM, Spear NE. Alcohol-mediated tactile conditioned aversions in infant rats: devaluation of conditioning through alcohol-sucrose associations. Neurobiol Learn Mem. 1996;66(2):121-132.

27. Clifton RK, Meyers WJ, Solomons G. Methodological problems in conditioning the headturning response of newborn infants. J Exp Child Psychol. 1972;13(1):29-42.

28. Amanzio M, Pollo A, Maggi G, Benedetti F. Response variability to analgesics: a role for non-specific activation of endogenous opioids. Pain. 2001;90(3):205-215.

29. Benedetti F, Maggi G, Lopiano L, et al. Open versus hidden medical treatments: the patient's knowledge about a therapy affects the therapy outcome. Prev Treat. 2003;6(1):article 1a. doi:10.1037/1522-3736.6.1.61a.

30. Colloca L, Lopiano L, Lanotte M, Benedetti F. Overt versus covert treatment for pain, anxiety, and Parkinson's disease. Lancet Neurol. 2004;3(11):679-684.

31. Schell AM, Dawson ME, Marinkovic K. Effects of potentially phobic conditioned stimuli on retention, reconditioning, and extinction of the conditioned skin conductance response. Psychophysiology. 1991;28(2): 140-153.

32. Rabin BM, Rabin JS. Acquisition of radiation- and lithium chlorideinduced conditioned taste aversion in anesthetized rats. Anim Learn Behav. 1984;12:439-441.

33. Pang R, Turndorf H, Quartermain D. Pavlovian fear conditioning in mice anesthetized with halothane. Physiol Behav. 1996;59(4-5):873-875.

34. Ghoneim MM, Chen P, el-Zahaby HM, Block RI. Ketamine: acquisition and retention of classically conditioned responses during treatment with large doses. Pharmacol Biochem Behav. 1994;49(4):1061-1066.

35. Bermudez-Rattoni F, Forthman DL, Sanchez MA, Perez JL, Garcia J. Odor and taste aversions conditioned in anesthetized rats. Behav Neurosci. 1988;102(5):726-732.

36. Ghoneim MM, Block RI, Fowles DC. No evidence of classical conditioning of electrodermal responses during anesthesia. Anesthesiology. 1992;76(5):682-688. 
37. Baeyens F, Eelen P, Van den Bergh O, Crombez G. Flavor-flavor and color-flavor conditioning in humans. Learn Motiv. 1990;21(4): 434-455.

38. Stevenson RJ, Boakes RA, Prescott J. Changes in odor sweetness resulting from implicit learning of a simultaneous odor-sweetness association: an example of learned synesthesia. Learn Motiv. 1998; 29(2):113-132.

39. Stevenson RJ, Prescott J, Boakes RA. The acquisition of taste properties by odors. Learn Motiv. 1995;26(4):433-455.

40. Lovibond PF, Shanks DR. The role of awareness in pavlovian conditioning: empirical evidence and theoretical implications. $J$ Exp Psychol Anim Behav Process. 2002;28(1):3-26.
41. Wickramasekera I. A conditioned response model of the placebo effect predictions from the model. Biofeedback Self Regul. 1980;5(1): $5-18$.

42. Benedetti F, Pollo A, Lopiano L, Lanotte M, Vighetti S, Rainero I. Conscious expectation and unconscious conditioning in analgesic, motor, and hormonal placebo/nocebo responses. J Neurosci. 2003; 23(10):4315-4323.

43. Finniss DG, Benedetti F. Mechanisms of the placebo response and their impact on clinical trials and clinical practice. Pain. 2005; 114(1-2):3-6.

44. Stewart-Williams S, Podd J. The placebo effect: dissolving the expectancy versus conditioning debate. Psychol Bull. 2004;130(2):324-340.

\section{Publish your work in this journal}

The Journal of Pain Research is an international, peer-reviewed, open access, online journal that welcomes laboratory and clinical findings in the fields of pain research and the prevention and managemen of pain. Original research, reviews, symposium reports, hypothesis formation and commentaries are all considered for publication.

\section{Dovepress}

The manuscript management system is completely online and includes a very quick and fair peer-review system, which is all easy to use. Visit http://www.dovepress.com/testimonials.php to read real quotes from published authors. 